\title{
Towards Non-destructive Burgers Vector Identification of Dislocations in Electronic Materials via Electron Channeling Contrast Imaging
}

\author{
R.J. Kamaladasa, ${ }^{*}$ M.E. Twigg, ${ }^{* *}$ M. De Graef, ${ }^{*}$ and Y.N. Picard* \\ *Materials Science and Engineering Department, Carnegie Mellon University, Pittsburgh, PA 15213 \\ **Electronics Science and Technology, Naval Research Laboratory, Washington, DC 20375
}

Transmission electron microscopy (TEM) has long been the standard method for dislocation analysis. TEM combines the necessary imaging resolution with the effective contrast mechanisms through electron diffraction in order to comprehensively identify the Burgers vector of individual dislocations. Both multi-beam dynamical diffraction simulations and invisibility criterion have long served as reliable avenues for TEM-based Burgers vector analysis.

However, a major limitation with TEM is the time-consuming, destructive sample preparation necessary for generating electron transparent specimens. Additionally, this required sample preparation greatly limits the viewable window available for analysis in a given specimen. The performance of many materials systems in electronic applications (thermoelectrics, photovoltaics, solid state lighting, etc.) are strongly limited by dislocations. However, these dislocations often exist in densities much too low $\left(<10^{6} / \mathrm{cm}^{2}\right)$ to be reliably captured in TEM specimens for subsequent analysis.

Scanning electron microscopy (SEM) has the significant advantage over TEM of being nondestructive and can rapidly image large areas. Diffraction based analysis of dislocations can be conducted inside a scanning electron microscope (SEM) by electron channeling contrast imaging (ECCI). Electron channeling is the phenomena where the backscattered electron yield is a strong function of the incoming electron beam trajectory relative to a crystalline sample orientation [1]. Local lattice bending by dislocations create local modulations $(\sim 3-5 \%)$ in the backscattered electron yield. The spatial character of a given intensity modulation will correspond to the Burgers vector of a specific dislocation present in the imaged sample surface. Since channeling contrast behavior is based on electron diffraction, ECCI can be implemented in manner similar to TEM for dislocation analysis.

With improved brightness and imaging resolution in modern SEM systems, ECCI shows promise for the imaging and identification of individual dislocations [2]. In this study, we demonstrate ECCI analysis of dislocations in single-crystalline material surfaces of interest in electronics applications: strontium titanate $\left(\mathrm{SrTiO}_{3}\right)$ and gallium nitride $(\mathrm{GaN})$. Evidence for invisibility criterion imaging is demonstrated for various sub-surface dislocations in (100) oriented $\mathrm{SrTiO}_{3}$ (Figure 1). We also show evidence for delineating threading screw versus threading edge dislocations in GaN (0001) surfaces by relating the spatial size of channeling contrast features with strain magnitude and surface relaxation effects. A simulation approach based on multi-beam dynamical diffraction theory [3] and developed for conical illumination is implemented to compute ECCI images of various defects in these material systems for comparison to experimental data. 
References

[1] D.G. Coates, Phil. Mag. 16 (1967) 1179.

[2] Y. N. Picard et al., Scripta Mater. 61 (2009) 773.

[3] M. De Graef, Intro. to Conventional Transmission Electron Microscopy, Cambridge Univ. Press (2003).

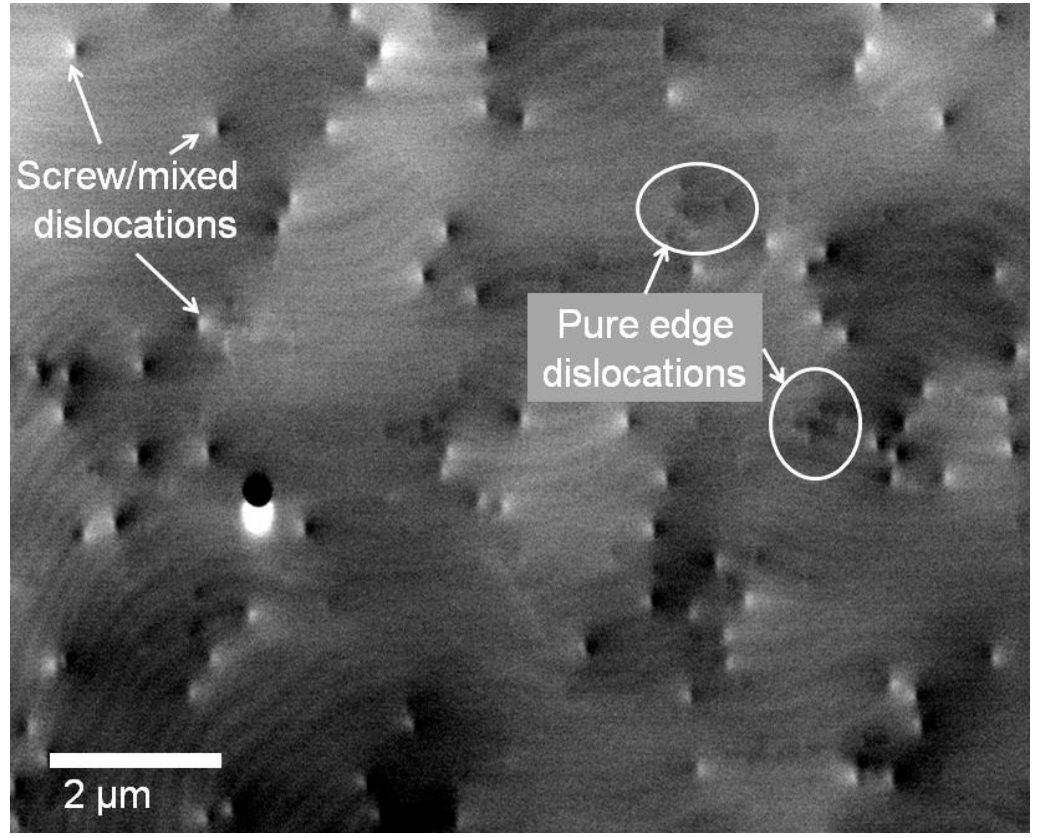

FIG. 1. ECCI micrograph of (0001) GaN recorded in a forescatter geometry with topographic enhancement sufficient to image atomic steps (curved lines) and channeling contrast features denoting various threading dislocations.
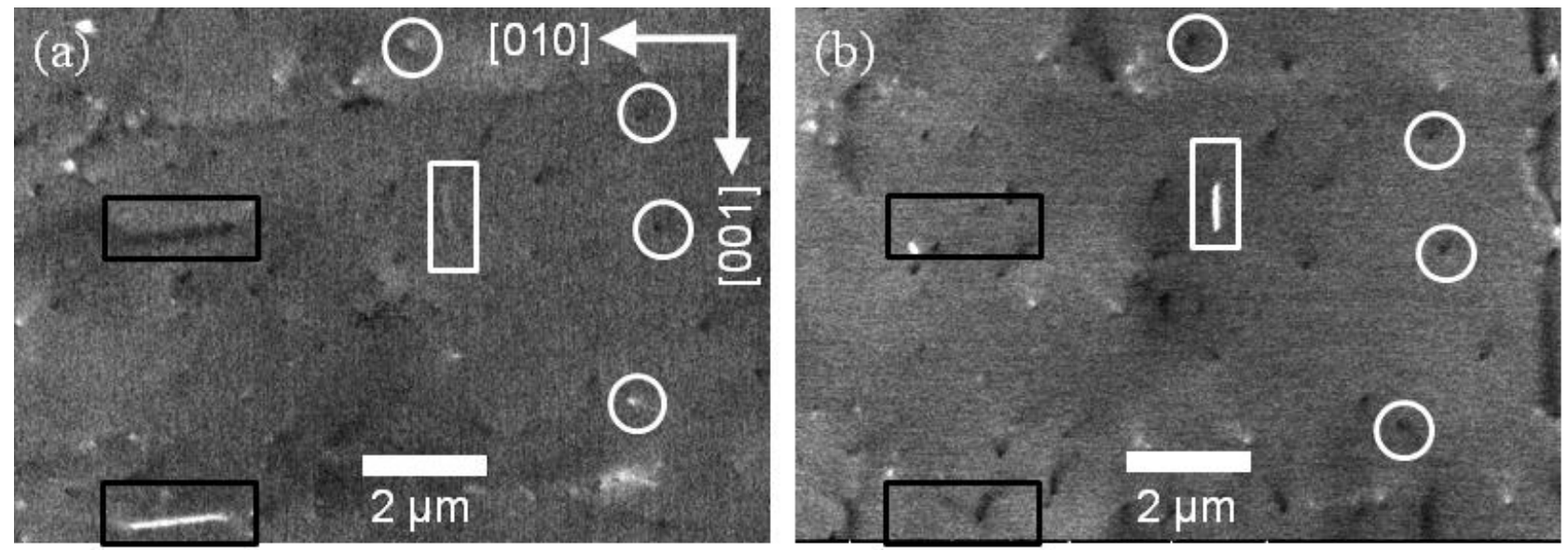

FIG. 2. ECCI micrographs of (100) $\mathrm{SrTiO}_{3}$ recorded in a backscatter geometry with (a) $\mathbf{g}=0-10$ and (b) $\mathbf{g}=001$. Dislocation segments visible in (a) and denoted by black rectangles are invisible in (b). A white rectangle denotes a dislocation line visible in (b) but with much weaker contrast in (a). Various dislocations penetrating the (100) surface are denoted by white circles. 